\title{
Fusion of OCT and hyperspectral imaging for tissue diagnosis and assessment
}

Gutiérrez, José, Real, Eusebio, Pardo, Arturo, LópezHiguera, José, Conde, Olga

José A. Gutiérrez, Eusebio Real, Arturo Pardo, José M. López-Higuera, Olga M. Conde, "Fusion of OCT and hyperspectral imaging for tissue diagnosis and assessment," Proc. SPIE 11362, Clinical Biophotonics, 1136207 (13 April 2020); doi: 10.1117/12.2555058

SPIE. Event: SPIE Photonics Europe, 2020, Online Only 


\title{
Fusion of OCT and hyperspectral imaging for tissue diagnosis and assessment
}

\author{
Jose A. Gutiérrez ${ }^{\mathrm{a}}$, Eusebio Real ${ }^{\mathrm{b}, \mathrm{c}}$, Arturo Pardo ${ }^{\mathrm{a}, \mathrm{b}}$, José M. López-Higuera ${ }^{\mathrm{a}, \mathrm{b}, \mathrm{c}}$, \\ Olga M. Conde ${ }^{* a, b, c}$ \\ a Photonics Engineering Group (GIF), TEISA department, University of Cantabria. Edificio I+D+I \\ Telecomunicación, Avda. Los Castros S/N, 39005 Santander, Cantabria, Spain; ' Instituto de \\ Investigación Sanitaria Valdecilla (IDIVAL), 39011 Santander, Cantabria, Spain ; ‘ Biomedical \\ Research Networking Center - Bioengineering, Biomaterials, and Nanomedicine (CIBER-BBN), \\ Av. Monforte de Lemos, 3-5. Pabellón 11. Planta 028029 Madrid
}

\begin{abstract}
The combination of molecular (hyperspectral imaging) and morphological (optical coherent tomography imaging) optical technologies helps in the assessment of biological tissue both in pathological diagnosis and in the follow-up treatments. The co-registration of both imaging features allows quantifying the presence of chromophores and the subsurface structure of tissue. This work proposes the fusion of two optical imaging technologies for the characterization of different types of tissues where the attenuation coefficient calculated from OCT imaging serves to track the presence of anomalies in the distribution of chromophores over the sample and therefore to diagnose pathological conditions. The performance of two customized hyperspectral imaging systems working in two complementary spectral ranges (VisNIR from 400 to $1000 \mathrm{~nm}$, and SWIR 1000 to $1700 \mathrm{~nm}$ ) and one commercial OCT system working at $1325 \mathrm{~nm}$ reveals the presence of fibrosis, collagen alterations and lipid content in cardiovascular tissues such as aortic walls (to assess on aneurysmal conditions) or tendinous chords (to diagnose the integrity of the valvular system) or in muscular diseases prone to fibrotic changes and inflammation.
\end{abstract}

Keywords: OCT, hyperspectral imaging, tissue chromophores, aortic wall, aneurysm.

\section{INTRODUCTION}

The aorta is the major blood vessel for transport of oxygenated blood from the heart to the arteries of the systemic circulation, and aortic diseases are a significant cause of death in developed countries [1]. The aorta is composed of three distinct structural layers: intima, media, and adventitia. The cellular and extracellular composition of these layers varies according to their functional performance. The intima layer is composed of a layer of endothelial cells that are in direct contact with blood. The media layer is composed of smooth muscle cells surrounded by an elastic extracellular matrix (ECM) of fibrous proteins, elastin, and collagen. Tensile strength for propulsion of the blood and elastic properties of the aorta are provided by elastin fibers. These fibers are arranged in concentric plates called elastic lamellae with a network of finer elastic fibers between lamellae. Collagen fibers are dispersed in the space between the lamellae and contribute to strengthen the aorta preserving its structural integrity. The outermost layer of the aorta is the adventitia that is composed by bundles of thick collagen fibrils and becomes surrounded by loose connective tissue. Under disease conditions, the fibrous structure and the content of collagen and elastin in the aortic ECM change [2]. Therefore, characterization of the molecular changes in ECM has a critical role in the diagnosis and severity assessment of different pathological conditions: aneurysm, dissection, atherosclerotic occlusion, and ageing-induced stiffening. Moreover, such characterization provides useful information to recognize pathophysiological mechanisms that underlie disease onset, progression, also helping clinical staff in the decision-making process during surgical interventions.

Different technologies have been applied to characterize aneurysmal tissue. FTIR (Fourier Transform Infrared) has been used to analyze biopsies of human ascending aortas [3-6] or porcine aortas [1] in terms of spectroscopic analysis searching for collagen and elastin content. In these applications, aortic tissue needs to be preprocessed into sections of $10 \mu \mathrm{m}$ thickness and measured in the $750-4000 \mathrm{~cm}^{-1}$ spectral range. This pretreatment and measurement modality of tissue is impossible in case of surgical decisions and other modalities more prone to interactive and real time conditions are required. In previous works [7] textural analysis of OCT (Optical Coherence Tomography) image sections of the media layer of the aortic wall revealed the anomalies present in the wall micro-structure. Also, in [8-9] image processing of OCT B-scans have allow

*olga.conde@unican.es; phone 34942 202238; fax 34942 200877; gif.teisa.unican.es

Clinical Biophotonics, edited by Daniel S. Elson, Sylvain Gioux, Brian W. Pogue, Proc. of SPIE Vol. 11362, 1136207 - @ 2020 SPIE · CCC code: 0277-786X/20/\$21 doi: $10.1117 / 12.2555058$ 
the accurate delineation of anomalous media layer regions where degradation appears as low intensity features in the OCT B-scans.

This work proposes the combination of OCT and custom built HSI (Hyperspectral Imaging) systems [10] to provide a diagnosis assessment in terms of morphological features provided by OCT and in terms of compositional features provided by HSI tracking the tissue composition as a measure of collagen, lipids, water and hemoglobin content employing affinity spectral metrics [11].

\section{MATERIALS AND METHODS}

\subsection{Tissue specimens}

In this viability study, human ex-vivo aortic specimens were obtained from the TAA (Thoracic Ascending Aorta) region after surgical interventions at the Marqués de Valdecilla University Hospital (Santander, Spain) under approval by "Comité Ético de Investigación Clínica de Cantabria” to INTRACARDIO project (code number 2018.008). Aortic specimens were divided for this study into two different categories: healthy donors in compliance with the standard heart donor criteria for control purposes (2 patients) and diseased aneurysm specimens (11 patients) from aneurysm surgical open repair interventions. Specimens were conserved in Phosphate Buffered Saline (PBS) and measured with the OCT system first and HSI systems afterwards within 12 hours from excision.

Once the human specimen is received at the laboratory, it is placed inside a customized 3D-printed fiducial grid (PLA, polylactic acid) for OCT and HSI co-registration purposes. The fiducial grid is divided into $9 \times 9$ individual squares or sections, coded (A to I) $\times\left(1\right.$ to 9 ), being each square size of $10 \times 10 \mathrm{~mm}^{2}$ as shown in Figure 1 . High spatial resolution OCT measurement is performed square by square. Just after finishing the whole OCT measurement, two HSI measurements (visNIR and SWIR) are performed in independent and single acquisitions for the whole specimen.

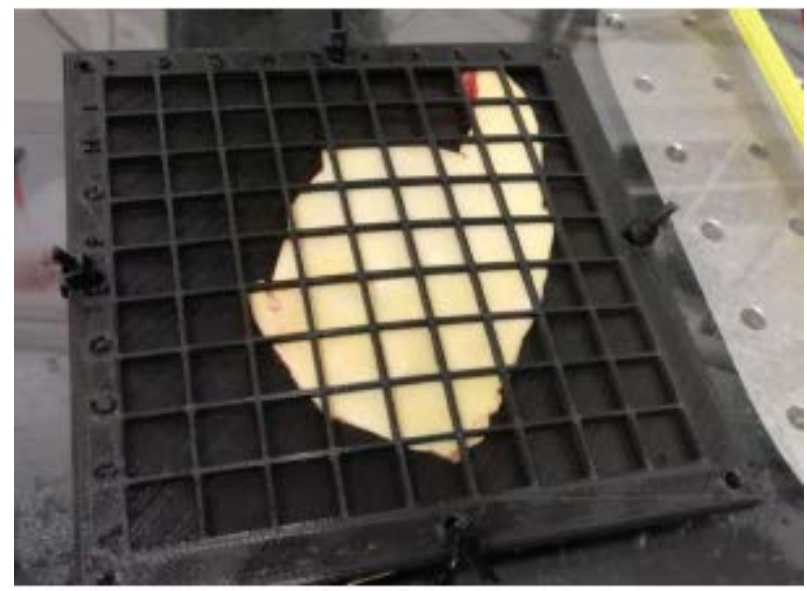

Figure 1. Aneurysmal specimen (id\#123) placement under fiducial grid before OCT and HSI measurements. Fiducial grid is composed of $9 \times 9$ squares with individual size of $10 \times 10 \mathrm{~mm}^{2}$.

\subsection{OCT and HSI optical systems}

For the Optical Coherence Tomography aortic tissue characterization [8], a bench-top OCT setup (OCS1300, Thorlabs, Newton, NJ, USA) is used. The light source is a swept source with center wavelength at $1325 \mathrm{~nm}$ and 100nm bandwidth at half maximum. Provided sensitivity is up to $100 \mathrm{~dB}$. The resolution of the system is of $12 \mu \mathrm{m}$ in depth and $25 \mu \mathrm{m}$ lateral, for a maximum B-scan dimension of $3 \mathrm{~mm}$ and $10 \mathrm{~mm}$, respectively. Resulting images are sampled with 512 pixels in depth by 1024 pixels lateral.

Two custom-built HSI (Hyperspectral Imaging) systems have been used for tissue spectroscopic characterization, one of them working in the visNIR (Visible Near InfraRed, 400-1000nm) range and the other in the SWIR (Short-Wave InfraRed, 1000-1700nm) range. Each system includes an embedded computer for storage and control [10]. The optical components 
of the visNIR-HSI system are a diffractive optics based on the ImSpector V10E spectrograph (Specim, Spectral Imaging Ltd., Finland) paired with a generic C-mount objective lens and a CMOS monochrome camera (Mako G-223B NIR, Allied Vision Technologies GmbH., Germany). In the case of the SWIR-HSI system: the ImSpector N17E spectrograph (Specim, Spectral Imaging Ltd., Finland), a generic C-mount objective lens and an InGaAs camera (Xeva-USB-FPA-1.7-320, Xenics Infrared Solutions, Belgium). One or two $500 \mathrm{~W}$ tungsten halogen bulbs are used as illumination sources for all measurements depending on the absorption characteristics of the sample. Relevant specifications of the HSI imaging systems are left in Table 1 . Hot pixels due to long exposure times were corrected via dark image subtraction as well as a spatial median filter of size 2.

Table 1. Specifications of the custom-built HSI systems in the visNIR and SWIR ranges.

\begin{tabular}{|l|l|l|}
\hline \multicolumn{1}{|c|}{ Specification } & \multicolumn{1}{|c|}{ visNIR system } & \multicolumn{1}{c|}{ SWIR system } \\
\hline Wavelength range & $400-1000 \mathrm{~nm}$ & 256 \\
\hline Spectral resolution & 218 & $1-50 \mathrm{~ms}$ \\
\hline Exposure range & $1-1000 \mathrm{~ms}$ & $320 \times 20.000$ \\
\hline Resolution (max.) & $1200 \times 1200$ & $5-50 \mathrm{~mm}, \mathrm{f} / 1.3$, var. aperture and zoom \\
\hline Focusing optics & $5-50 \mathrm{~mm}, \mathrm{f} / 1.3$, var. aperture and zoom & \\
\hline
\end{tabular}

\subsection{OCT characterization of aortic tissue}

Aortic walls have been measured with the OCT system providing C-scans of dimensions $10 \times 10 \mathrm{~mm}^{2}$ lateral and $3 / n \mathrm{~mm}$ in depth, where $n$ is the refractive index of the cardiovascular tissue, with a value of 1.38 at $1300 \mathrm{~nm}[8,12]$. In terms of resolution, this implies a final $4.24 \mu \mathrm{m} / \mathrm{pixel}$ axial resolution and $9.76 \mu \mathrm{m} / \mathrm{pixel}$ lateral resolution. Intensity OCT is converted to depth-resolved attenuation coefficient, $\mu_{t}$, according to Equation 1 presented by Vermeer et al. [13].

$$
\mu_{t}=\frac{I[i]}{2 \Delta \sum_{k=i+1}^{\infty} I[k]},
$$

where $\Delta$ represents pixel dimensions in microns, $I[i]$ represents the $\mathrm{i}^{\text {th }}$ pixel in depth and the summation comprehends the rest of the pixels in depth. At the central wavelength of the OCT, the attenuation coefficient $\mu_{t}$, can be considered mainly due to the scattering component. Figure 2 shows the attenuation coefficient (Fig.2c) calculated from the intensity OCT Ascans values (Fig. 2b) on two different regions of interest (ROIs: red and green) of one section (fiducial grid square) of a pathological specimen (Fig. 2a). On the graphs (Fig. 2b and Fig. 2c), light color lines display the value of the parameter (OCT intensity and attenuation coefficient respectively) for each individual pixel of the ROI, and dark color lines represent the average of the parameter for each ROI. Aortic attenuation coefficient varies in the range $10-60 \mathrm{~cm}^{-1}$ which agrees with literature [14]. This localized coefficient enables tissue characterization based on attenuation estimates from OCT data.

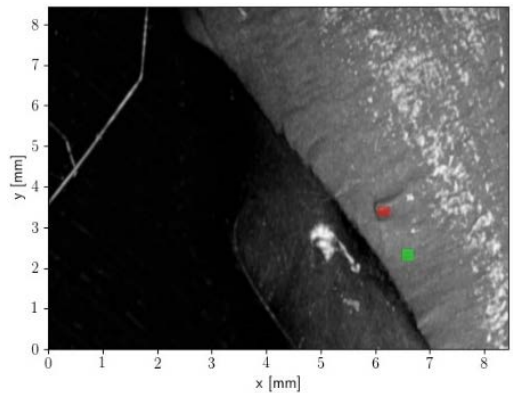

(a)
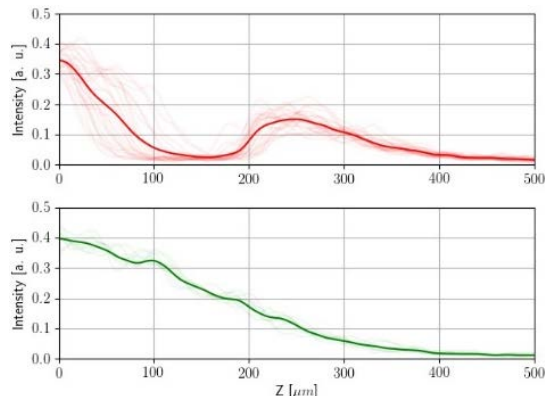

(b)

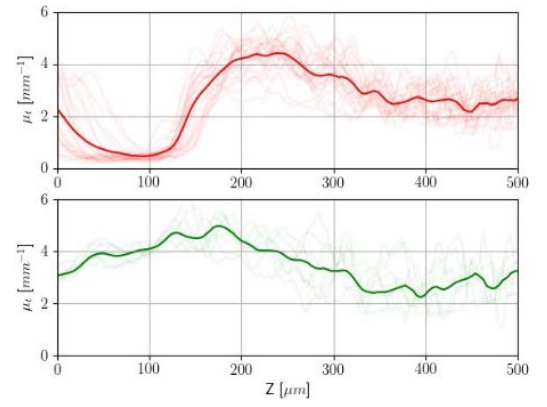

(c)

Figure 2. OCT intensity and attenuation coefficient for a pathological specimen, id\#130-C4: (a) section of a fiducial grid square with two different regions of interest (ROI, red and green); (b) OCT intensity for each ROI, dark color lines represent the average OCT intensity for the whole ROI and light color lines display each individual A-scan within the ROI; (c) attenuation coefficient for each ROI, dark color lines represent the average attenuation coefficient for the whole ROI, and light color lines display the attenuation coefficient for each pixel within the ROI. 
The attenuation coefficient $\mu_{t}$ is then used to segment, to identify and to visualize the presence of sub-surface aortic tissue perturbations in terms of spatial maps: (1) the distribution of MIP (maximum projection) of attenuation coefficient (Fig. 3a); (2) the distribution of $m I P$ (minimum projection) of attenuation coefficient (Fig. 3b); (3) the inverse value of $m I P$ to enhance the presence of a reduction of the attenuation coefficient (Fig. 3c) and (4) the value of the axial depth, $z_{\min }$, where the lowest value of the attenuation coefficient appears (Fig. 3d).

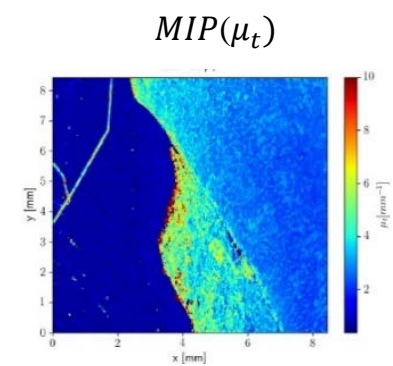

(a)

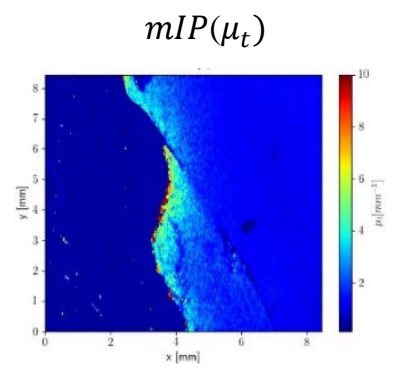

(b)
Inverse of $m I P\left(\mu_{t}\right)$

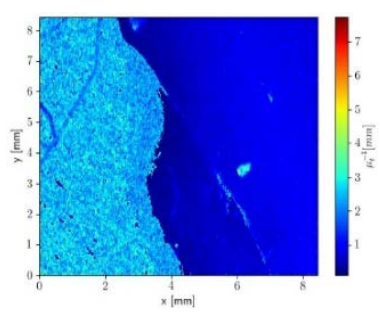

(c)

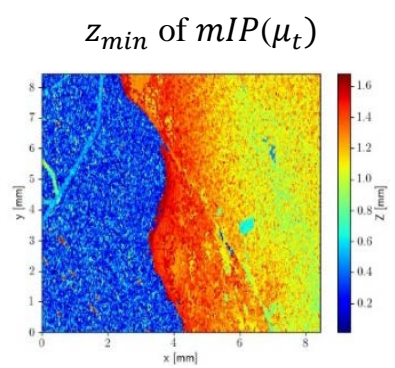

(d)

Figure 3. Spatial maps of the distribution of metrics of the attenuation coefficient for id\#130-C4: (a) maximum value of the attenuation coefficient; (b) minimum value of the attenuation coefficient; (c) enhancement of the reduction of the attenuation coefficient and (d) axial depth where the lowest value of the attenuation coefficient appears.

\subsection{HSI characterization of aortic tissue}

After OCT measurement, spectroscopic aortic tissue features are obtained in terms of hyperspectral images. Radiation from tissue is converted in reflectance images performing spectral calibration against a standard white reference material located at the same distance and lighting conditions. Reflectance $R(\lambda)$ was calculated with the well-known equation (2):

$$
R(\lambda)=\frac{I_{s}(\lambda)-I_{b g}(\lambda)}{I_{w}(\lambda)-I_{b g}(\lambda)},
$$

where $I_{s}(\lambda)$ is the sample re-emitted intensity measurement, $I_{w}(\lambda)$ is a diffuse reflectance reference standard under identical illumination conditions, and $I_{b g}(\lambda)$ is the background intensity due to electronic noise in the camera sensor. Spectral calibration is achieved through linear least-squares on a set of 3 known absorption peaks from a Spectralon Wavelength Calibration Standard (WCS) namely WCS-MC-020 (Labsphere, Inc., New Hampshire, USA). Reflectance $R(\lambda)$ is converted into absorbance through equation (3):

$$
\operatorname{Abs}(\lambda)=10 \log _{10}\left(\frac{1}{R(\lambda)}\right)
$$

As stated, different components or chromophores can be found on the aortic tissue. Its presence or over-expression can be tracked by computing spectral affinity metrics or spectral similarity against known chromophore spectral references. In this work the presence of five tissue chromophores is assessed [15-16]: oxygenated and deoxygenated hemoglobin, collagen, lipid, and water content. Figure 4 displays the spectral fingerprints of the evaluated reference chromophores in both spectral ranges: visNIR (green shadowed background) and SWIR (red shadowed background).

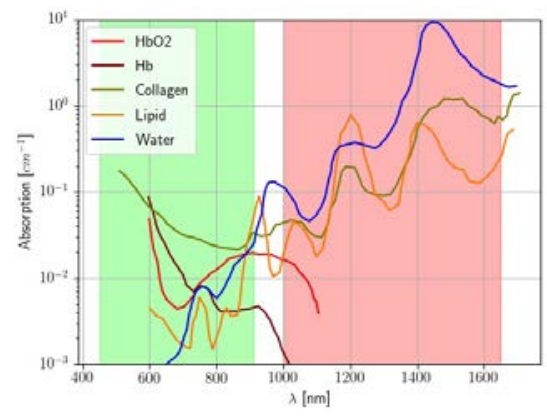

Figure 4. Spectral features of the main chromophores in aortic tissue [15-16]: deoxygenated hemoglobin (brown), oxygenated hemoglobin (red), collagen (green), lipid (orange) and water (blue). 
The discrimination of chromophores presence is based on the comparison of the measured spectra with the reference one. This comparison is done using a metric of similarity as a criterion. For the similarity calculation, deterministic or stochastic techniques can be applied. Deterministic techniques are based on distance, angle, or correlation between spectra. Stochastic metrics use sample properties and develop spectral criteria, such as divergence or probability, to measure dissimilarity between two spectra. Being $x\left(x_{1}, \cdots, x_{n}\right)$ the specimen spectrum to be evaluated, $y\left(y_{1}, \cdots, y_{n}\right)$ the chromophore known spectrum and $n$ is the number of spectral bands, three similarity metrics have been evaluated:

- Euclidean Distance (ED): it measures the spectral distance between two fingerprints. ED equals 0 when both spectral fingerprints are identical and increases as they differ:

$$
E D(x, y)=\sqrt{\sum_{i=1}^{n}\left(x_{i}-y_{i}\right)^{2}}
$$

- $\quad$ Bray-Curtis similarity (BCS) [17]: it is not a distance since it does not satisfy triangle inequality. The Bray-Curtis Similarity is calculated as a percentage, it is 0 when both spectral fingerprints are completely different and 100 when they are identical:

$$
\operatorname{BCS}(x, y)=100-\left(\frac{\sum_{i=1}^{n}\left|\left(x_{i}-y_{i}\right)\right|}{\sum_{i=1}^{n} x_{i}+\sum_{i=1}^{n} y_{i}}\right) * 100
$$

- $\quad$ Spectral information divergence (SID) [18-19]: this metric model the spectral band-to-band variability as a result of uncertainty caused by randomness. SID is derived from divergence theory and calculates the probabilistic behaviors between spectral signatures:

$$
\begin{gathered}
S I D(x, y)=D(x \| y)+D(y \| x) \\
D(x \| y)=\sum_{i=1}^{n} p_{i} D_{i}(x \| y)=\sum_{i=1}^{n} p_{i}\left(I_{i}(y)-I_{i}(x)\right)=\sum_{i=1}^{n} p_{i} \log _{2}\left(\frac{p_{i}}{q_{i}}\right) \\
D(y \| x)=\sum_{i=1}^{n} q_{i} D_{i}(y \| x)=\sum_{i=1}^{n} q_{i}\left(I_{i}(x)-I_{i}(y)\right)=\sum_{i=1}^{n} q_{i} \log _{2}\left(\frac{q_{i}}{p_{i}}\right)
\end{gathered}
$$

where $D(x \| y)$ and $D(y \| x)$ are called the relative entropy of $y$ with respect to $x$ and $x$ with respect to $y$ respectively and are also known as the Kullback-Leibler information measures. $D_{i}$ is the relative entropy with respect to band $i$. The probability vectors $p\left(p_{1}, \cdots, p_{n}\right)$ and $q\left(q_{1}, \cdots, q_{n}\right)$ for the spectral signatures $x$ and $y$ are defined as $p_{i}=\frac{x_{i}}{\sum_{m=1}^{n} x_{m}}$ and $q_{i}=\frac{y_{i}}{\sum_{m=1}^{n} y_{m}}$. The self-information measures denoted by $I_{i}(x)$ and $I_{i}(y)$ for spectral signatures $x$ and $y$ for band $i$ can be defined as $I_{i}(x)=-\log _{2}\left(p_{i}\right)$ and $I_{i}(y)=-\log _{2}\left(q_{i}\right)$ respectively.

\section{RESULTS AND DISCUSSION}

As discussed in previous works [9], aortic anomalies under aneurysm pathology appear as a non-continuous progression of the cardiovascular tissue degeneration. OCT helped in the diagnose of anomalous regions through the segmentation of deeper tissue areas where the OCT intensity of the B-scans suddenly had decreased. These areas underwent changes in their refractive index due to morphological and compositional alterations. However, that approach was sensitive to noise measurement artifacts within the A-scan profile. Here, a new OCT tissue characterization is proposed based on the estimation of the attenuation coefficient of the aortic wall. Figure 5a shows the white light photography of an aneurysmal specimen and the OCT spatial resolution maps corresponding to the areas where the attenuation coefficient $\mu_{t}$ decreases at specific points in tissue and the axial depth where this reduction appears. Figures $5 \mathrm{~b}$ and $5 \mathrm{c}$ are the result of mosaicking the 29 OCT measurement sections occupied by the specimen within the fiducial grid. Figure $5 \mathrm{~b}$ represents the inverse of the minimum value of the attenuation coefficient that implies a significant change in tissue morphology. Figure 5c shows the spatial distribution map of axial depths, $z_{\min }$, where the attenuation coefficient achieved its minimum $m I P\left(\mu_{t}\right)$. In this last figure, values over $1.2 \mathrm{~mm}$ implies that the minimum of the attenuation coefficient is attained due to signal absorption as light travels deeper in tissue and it has no diagnostic implications. 


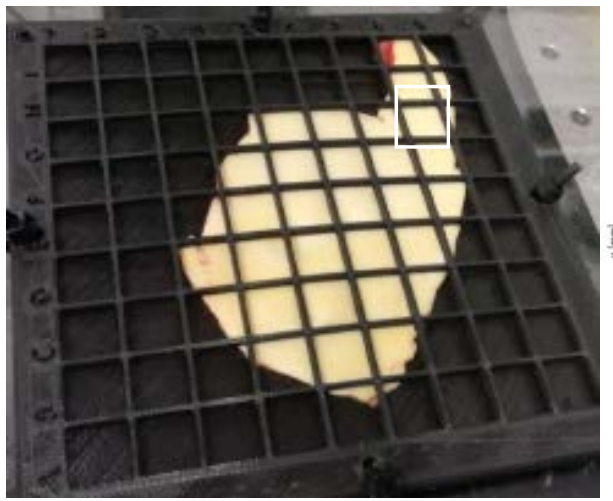

(a)

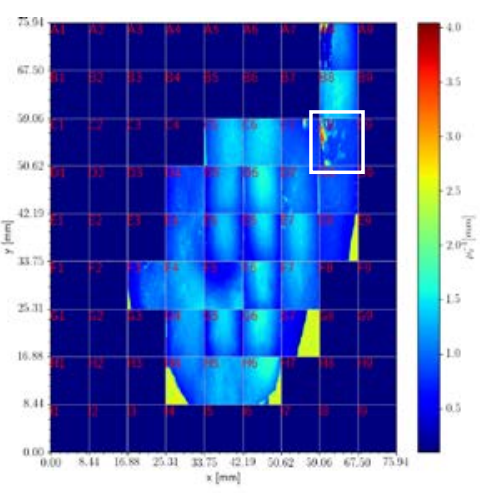

(b)

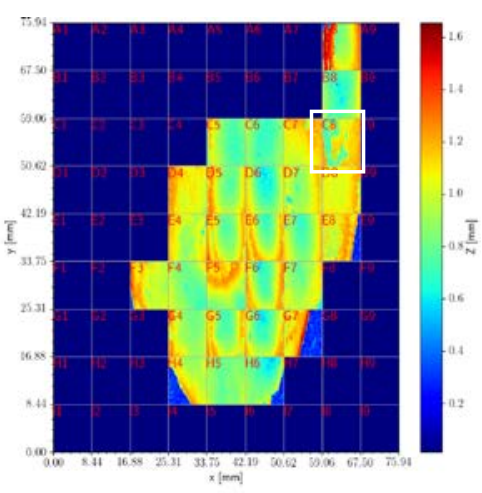

(c)

Figure 5. Pathological specimen, id\#123: (a) white light photography; (b) inverse on the minimum value of the attenuation coefficient; (c) distribution map of axial depths $Z_{\min }$ where the lowest value of the attenuation coefficient appears.

When observed closely, section C8 of Fig. 5b shows that a contrast appears the map of the inverse of the minimum of $\mu_{t}$. Fig. 5c reveals that this minimum value is located at around $700 \mu \mathrm{m}$ below tissue surface, being located within the aortic wall media layer. Figure 6 shows a close-up of this C8 section. Apart from the expected different spatial resolution of both modalities, Figure 6 shows that in this area appears an uneven distribution of the hemoglobin content with a similarity metric even higher than that of collagen. Due to low number of spectral points of the hemoglobin spectra in the SWIR range (Figure 4), the hemoglobin content could be here overestimated. Lipid is the chromophore with the smaller presence, the same happens with water that may only reveal the hydration status of the sample due to its previous immersion in PBS.

Collagen

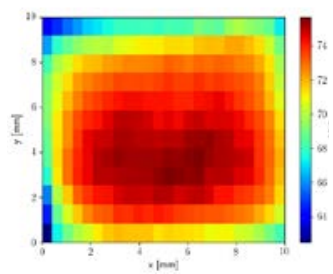

$\operatorname{MIP}\left(\mu_{t}\right)$

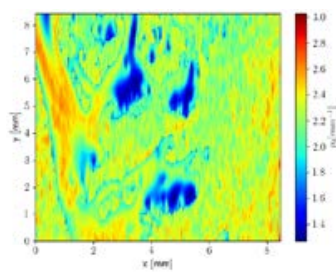

$\mathrm{Hb}$

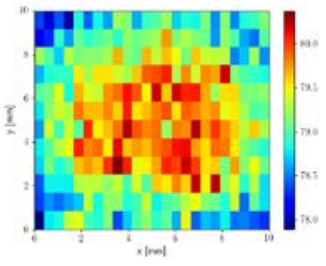

$m I P\left(\mu_{t}\right)$

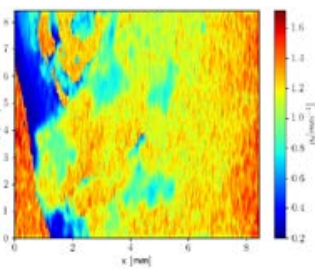

$\mathrm{HbO}_{2}$

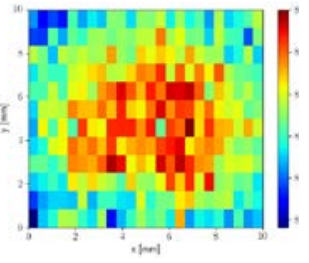

Inverse of $m I P\left(\mu_{t}\right)$

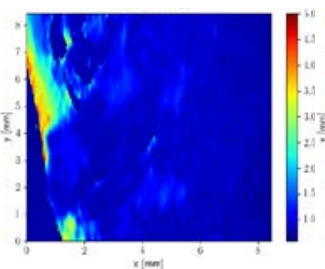

Lipid

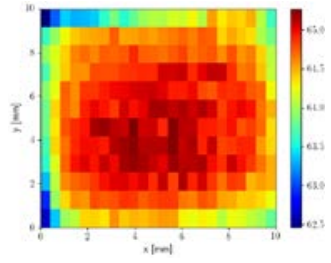

$z_{\min }$ of $\operatorname{mIP}\left(\mu_{t}\right)$

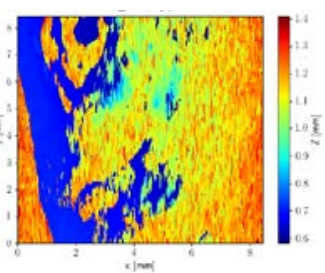

Figure 6. Pathological specimen, id\#123-C8: (upper row) similarity metric BCS in the SWIR-HSI range when tracking collagen, deoxygenated hemoglobin, oxygenated hemoglobin, lipid content and water content; (lower row) contrast maps provided by the attenuation coefficient from OCT.

Figure 7 shows other section from another aneurysmal specimen of a different patient. As before, a contrast map is perceived in the inverse of the minimum attenuation coefficient. In the collagen map can be appreciate a texture pattern that appear to coarsely correlate with the inverse of $m I P\left(\mu_{t}\right)$. Again, water and lipid are less expressed, and hemoglobin appears also in a high degree. 
Collagen

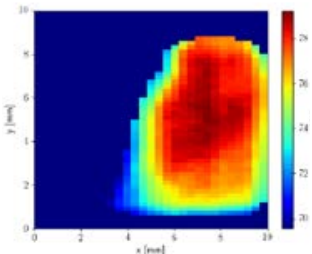

$\operatorname{MIP}\left(\mu_{t}\right)$

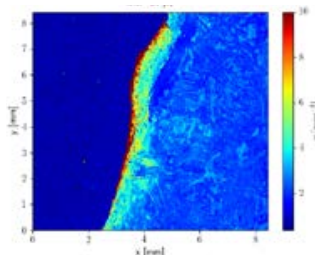

$\mathrm{Hb}$

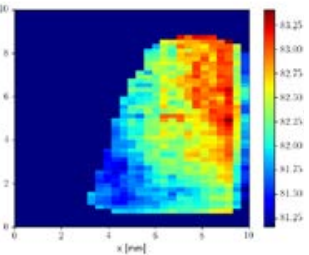

$m I P\left(\mu_{t}\right)$

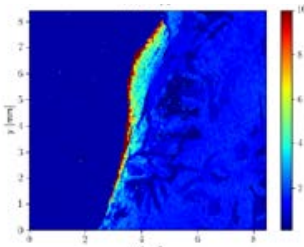

$\mathrm{HbO}_{2}$

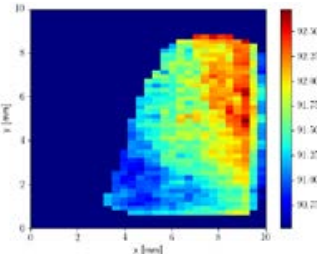

Inverse of $m I P\left(\mu_{t}\right)$

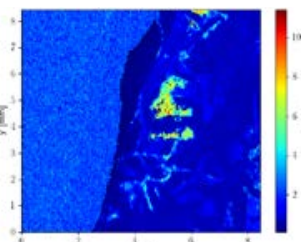

$\sin 1$
Lipid

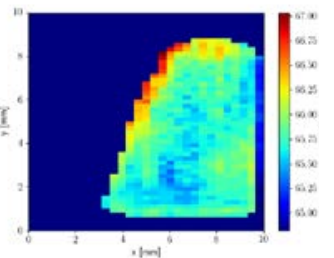

$z_{\text {min }}$ of $m I P\left(\mu_{t}\right)$

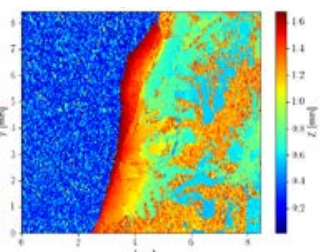

Figure 7. Pathological specimen, id\#131-C4: (upper row) similarity metric BCS in the SWIR-HSI range when tracking collagen, deoxygenated hemoglobin, oxygenated hemoglobin, lipid content and water content; (lower row) contrast maps provided by the attenuation coefficient from OCT.

Finally, Figure 8 shows a section from a control specimen. The left part of the section corresponds with the basis of the fiducial grid. Tissue is located on the right half part of the section. In a healthy specimen, both the maximum and the minimum value of the attenuation coefficient provide a smooth and continuous spatial pattern. The similarity metric for the collagen content has increased between $7 \%-11 \%$ respect aneurysmal specimens and it presents also a quasi-uniform distribution [3]. Hemoglobin content has slightly decreased, and water and lipids remain in the same scale.

Collagen

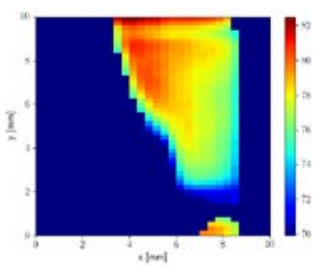

$\operatorname{MIP}\left(\mu_{t}\right)$

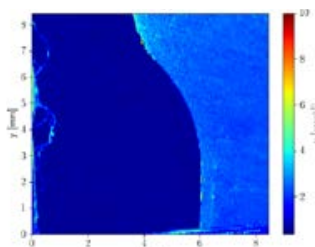

$+\infty=1$
$\mathrm{Hb}$

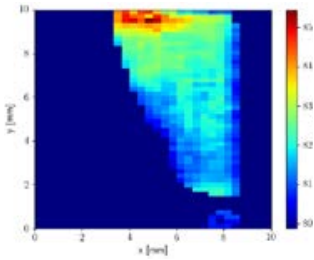

$m I P\left(\mu_{t}\right)$

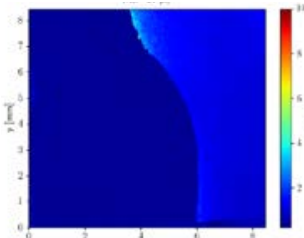

$\ln 1$
$\mathrm{HbO}_{2}$

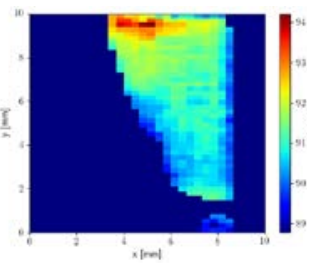

Inverse of $m I P\left(\mu_{t}\right)$

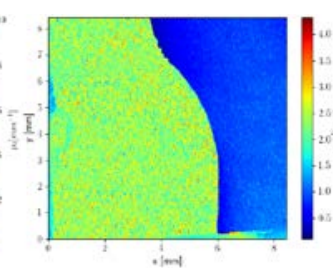

Lipid

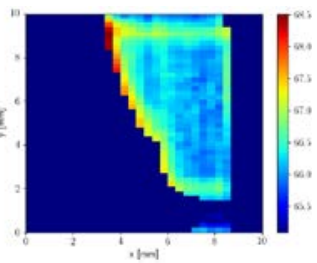

$z_{\min }$ of $m I P\left(\mu_{t}\right)$

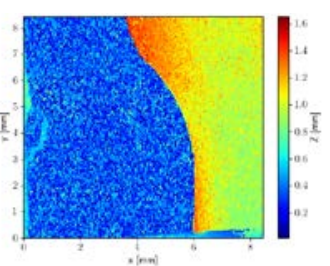

Figure 8. Control specimen, id\#129-C5: (upper row) similarity metric BCS in the SWIR-HSI range when tracking collagen, deoxygenated hemoglobin, oxygenated hemoglobin, lipid content and water content; (lower row) contrast maps provided by the attenuation coefficient from OCT.

\section{CONCLUSIONS}

In this viability study, a fusion of OCT and HSI modalities has been proposed to develop visual assessment of morphological and compositional status of human cardiovascular tissue from the thoracic ascending aorta when affected by aneurysm pathology. The main advantage of both modalities is that no tissue pretreatment is required, neither tissue 
preparation in terms of thin slides nor tissue cryosection. This favor technology translation to the operating theatre. OCT provides detailed and high spatial resolution of tissue. HSI is performed with custom-built systems in the visNIR and SWIR ranges providing moderate spatial resolution but with an enhanced time performance. OCT intensity depth profiles are used to estimate the value of tissue attenuation coefficient that, as a physical magnitude, provides segmentation values independent of the OCT intensity values used for representation. This can lead to depth resolved tissue composition identification. The high resolution and inherent contrast of OCT is applied to track spectroscopic changes and to compare and evaluate the chromophore presence (collagen, hemoglobin, lipid, water) under an oriented focus. Future work will be aimed to evaluate the whole set of spectral similarity metrics, in visNIR and SWIR spectral ranges, combining them to take profit of the different approaches for the calculation of the similarity criteria.

\section{Acknowledgements}

The authors would like to thank clinical staff (Marta Calvo, Alejandro Pontón, José Francisco Gutiérrez and Juan Francisco Nistal) from the Cardiovascular Surgery Service at the Hospital Universitario Marqués de Valdecilla for their clinical support and assistance providing the aortic human specimens.

\section{Funding}

Spanish Ministry of Science, Innovation and Universities (FIS2010-19860, TEC2016-76021-C2-2-R), Spanish Ministry of Economy, Industry and Competitiveness and Instituto de Salud Carlos III (DTS17-00055, DTS15- 00238), Instituto de Investigación Valdecilla (INNVAL16/02, INNVAL18/23) and Spanish Ministry of Education, Culture, and Sports (FPU16/05705).

\section{REFERENCES}

[1] Cheheltani, R., McGoverin, C. M., Rao, J., Vorp, D. A., Kiani, M. F., \& Pleshko, N. "Fourier transform infrared spectroscopy to quantify collagen and elastin in an in vitro model of extracellular matrix degradation in aorta." The Analyst, 139(12), 3039-3047 (2014).

[2] Tsamis, A., Krawiec, J. T., \& Vorp, D. A., "Elastin and collagen fibre microstructure of the human aorta in ageing and disease: a review," Journal of the Royal Society, Interface, 10(83), 20121004. (2013).

[3] Bonnier F, Rubin S, Ventéo L, Krishna CM, Pluot M, Baehrel B, Manfait M, Sockalingum GD., "In-vitro analysis of normal and aneurismal human ascending aortic tissues using FT-IR microspectroscopy," Biochim Biophys Acta, 1758(7), 968-73 (2006).

[4] Rubin S, Bonnier F, Sandt C, Ventéo L, Pluot M, Baehrel B, Manfait M, Sockalingum GD.,”Analysis of structural changes in normal and aneurismal human aortic tissues using FTIR microscopy,” Biopolymers, 89(2), 160-9 (2008).

[5] Bonnier F, Rubin S, Debelle L, Ventéo L, Pluot M, Baehrel B, Manfait M, Sockalingum GD, "FTIR protein secondary structure analysis of human ascending aortic tissues,” J Biophotonics, 1(3), 204-14 (2008).

[6] Bonnier F, Bertrand D, Rubin S, Ventéo L, Pluot M, Baehrel B, Manfait M, Sockalingum GD. "Detection of pathological aortic tissues by infrared multispectral imaging and chemometrics," Analyst, 133(6), 784-90 (2008).

[7] Real, E., Eguizábal, A., Pontón, A., Díez, M.C., Val-Bernal, J.F., Mayorga, M., Revuelta, J.M., López-Higuera, J.M., Conde, O.M., "Optical coherence tomography assessment of vessel wall degradation in thoracic aortic aneurysms,” J. Biomed. Opt. 18(12), 126003 (2013).

[8] Real, E., Eguizábal, A., Pontón, A., Díez, M.C., Val-Bernal, J.F., Mayorga, M., Revuelta, J.M., López-Higuera, J.M., Conde, O.M., "Identification of vessel wall degradation in ascending thoracic aortic aneurysms with OCT," Biomed. Opt. Express 5(11), 4089-4100 (2014).

[9] Real, E., Eguizábal, A., Pontón, A., Díez, M.C., Val-Bernal, J.F., Mayorga, M., Revuelta, J.M., López-Higuera, J.M., Conde, O.M., "Hessian analysis for the delineation of amorphous anomalies in optical coherence tomography images of the aortic wall,” Biomed. Opt. Express 7(3), 1415-1429 (2016).

[10] Gutiérrez-Gutiérrez, J.A., Pardo, A., Real, E., López-Higuera, J.M., Conde, O.M., "Custom Scanning Hyperspectral Imaging System for Biomedical Applications: Modeling, Benchmarking and Specifications," Sensors 19, 1692 (2019). 
[11] Pardo, A., Gutiérrez-Gutiérrez, J.A., López-Higuera, J.M., Conde, O.M., "Context-free hyperspectral image enhancement for wide-field optical biomarker visualization,” Biomed. Opt. Express 11(1), 133-148 (2020).

[12] van der Meer, F.J., Faber, D.J., Cilesiz, I., van Gemert, M.J.C., van Leeuwen, T.G., "Temperature-dependent optical properties of individual vascular wall components measured by optical coherence tomography,” J. Biomed. Opt., 11(4), 41120 (2006).

[13] Vermeer, K. A., Mo, J., Weda, J. J. A., Lemij, H. G. and de Boer, J. F., "Depth-resolved model-based reconstruction of attenuation coefficients in optical coherence tomograph,” Biomed Opt Express 5(1), 322-337 (2014).

[14] Regar, E., van Leeuwen, T.G., Serruys, P.W., [Optical Coherence Tomography in Cardiovascular Research], Abingdon: Informa Healthcare (2007).

[15] Prahl S. Optical absorption of hemoglobin. 1999. http://omlc.ogi.edu/spectra/hemoglobin/index.html (18 November 2019).

[16] Sekar, K.V., Pagliazzi, S., Negredo, M., Martelli, E., Farina, F., Dalla Mora, A., Lindner, A., Farzam, C., PérezÁlvarez, P., Puig, N., Taroni, P., Pifferi, A., Durduran, T, "In Vivo, Non-Invasive Characterization of Human Bone by Hybrid Broadband (600-1200 nm) Diffuse Optical and Correlation Spectroscopies, " PloS one, 11(12), e0168426 (2016).

[17]Bray, J. R., Curtis, J.T., “An ordination of upland forest communities of southern Wisconsin,” Ecological Monographs, 27, :325-349 (1957).

[18] Seshasai, M., Prasad, K., Venkateswaran, K., Ramana, K., Dwivedi, R., Parth. R., "A new hybrid spectral similarity measure for discrimination of Vigna species,” Intl. Journal of Remote Sensing, 32, 4041-4053 (2011).

[19]Chang, C.I, "An information-theoretic approach to spectral variability, similarity, and discrimination for hyperspectral image analysis," IEEE Transactions on Information Theory, 46(5), 1927-1932 (2000). 